\title{
The future of pharmacology
}

\author{
${ }^{*, 1}$ Patrick Vallance \& ${ }^{2}$ Trevor G. Smart
}

${ }^{1}$ Centre for Clinical Pharmacology, Division of Medicine, UCL, 5 University Street, London, WC1E 6JJ and ${ }^{2}$ Department of Pharmacology, UCL, Gower Street, London WC1E 6BT

British Journal of Pharmacology (2006) 147, S304-S307. doi:10.1038/sj.bjp.0706454

Keywords: Pharmacology; clinical pharmacology; perspective

Correct attribution is an important part of science and allows us to understand the evolution of ideas and discovery. So it is with some shame that we start this article with a quote that comes from Niels Bohr but may have originated with Mark Twain 'Prediction is very difficult especially about the future'. The task in trying to write an article about the future of pharmacology is made more difficult by Lao Tzo, a poet from 6th century China who declared that "Those who have knowledge don't predict, and those who predict don't have knowledge".

Looking back, the use of herbal and animal extracts and minerals has been with us for thousands of years as a very early form of therapeutics. Indeed, Cladius Galen could be considered as one of the first practitioners of pharmacology as early as 150AD when he recognised the importance of experimentation and theory in the rational use of medicines (Galen on Pharmacology). The Swiss physician, Paracelsus, provided the early momentum for pharmacology by investigating the active principles/ingredients of many medieval preparations. It was he who recognised that all drugs can be poisons and acknowledged that the dose alone determines whether or not a compound is therapeutically useful or toxic, possibly the earliest recognition of the therapeutic window, a feature that will be familiar to today's health care practitioners. In essence though, the science of pharmacology that is broadly recognised now emerged in the mid 19th century with Oswald Schmiedeberg who also founded the very first journal of pharmacology (see also Cuthbert, this issue). The fundamental concepts of pharmacology arrived during this formative time with others developing the concepts of 'the drug receptor', structure-function relationships for drug molecules, and A.J. Clark (one of our (T.G.S.) predecessors at UCL) was the first to apply the Law of Mass Action to drug receptor operation (see also Rang, this issue). The driving force in pharmacology was to understand, quantitate and document the effects of substances on whole-body physiology. Never just what does extract $\mathrm{X}$ do, but also how does it do it and what is the active ingredient. The two strands of this approach subsequently developed in academia and industry. The concept of the concentration-response relationship has been central to pharmacology and of course remains central to the practice of medicine. Within the pharmaceutical industry the partnership of chemist and pharmacologist has been key. Within academia the role of the chemist has often been less evident and the links between pharmacology and physiology have been key - the

*Author for correspondence; E-mail: p.vallance@ucl.ac.uk pharmacologist using small molecular probes to perturb physiology and thereby elucidate mechanisms. Through careful identification of mechanisms and the development of chemicals that interfere with specific pathways, the introduction of powerful new medicines over the past 50 or so years has meant that doctors can now offer specific and effective treatments across a wide range of diseases.

So where are we now? A brief history of pharmacology on the website of the American Chemical Society (Scheindlin, 2001) ends its historical introduction noting that 'today there is a pharmacology department in every college of medicine or pharmacy'. Not so, at least in the U.K. Departments of Pharmacology have disappeared from many universities, and many medical schools no longer teach or examine specifically in the area of pharmacology or clinical pharmacology. The vogue is for crosscutting themes. It just so happens that the cut crosses fashionably in a different direction at the moment, across disciplines like physiology, biochemistry and pharmacology and into organ-based groupings or more fashionable 'omics'. For current research perhaps this matters less since we all attempt to use multiple techniques to address the question in hand. For teaching, training and the future it might matter a very great deal. At the fundamental science end, new drug discovery requires a thriving academic base in pharmacology, and at the clinical end over $60 \%$ of the elderly take at least one prescription medicine per week, about 650 million prescriptions are issued on the NHS each year (ABPI, 2005) and many patients admitted to a medical ward take 10 or more medicines. Medicines of course do not respect the boundaries of organ systems, either in their wanted effects or their unwanted effects. Indeed, it is estimated that $5-10 \%$ of all hospital admissions are due to adverse drug reactions (Pirmohamed et al., 2004). Pharmacological approaches to treatment seem unlikely to be on the wane and the need for experts in all aspects of drug action has never been greater. In this article, we have tried to identify some of the themes that may be important for the development of pharmacology.

\section{Research}

Bioassay has been an essential tool, and one only has to read the contributions in this special edition of the British Journal of Pharmacology to see the impact it has had. In one of the main areas of research of one of us (P.V.) (endothelial biology and nitric oxide (see also Moncada \& Higgs, this issue)) the pivotal paper was a simple functional assessment of the relaxation of a blood vessel, showing that an intact endothelial 
layer was essential for the relaxant response to acetylcholine (Furchgott \& Zawadzki, 1980). No fancy techniques, but an inventiveness in the approach to identify the mediator involved. Bioassay and assessment of functional approach seems set to remain a core activity for pharmacologists and may be entering a new golden age. The generation of knockout animals has almost become routine, and approaches such as gene silencing through small interfering RNA, present highly targeted routes to explore the function of individual proteins, whether they are receptors, enzymes, ion channels or intracellular signalling molecules. However, the challenge for many of these molecular approaches lies in the methods used to assess the phenotype. Hence the many recent initiatives in 'integrative physiology' or 'functional genomics'. These are core skills of the pharmacologist. Add to this, the increasing need to supplement genetic approaches with small molecular probes to disrupt the pathways - the emerging field of chemical biology - and it all sounds rather familiar. The genetic or genomic approach often has either an all-or-none signal, or perhaps a three point dose-response curve in knockout mice of $-/-,-/+$ and $+/+$, whereas the small molecular inhibitor allows construction of the entire relationship and at varying time points. Proving causality needs the selective interruption of specific pathways, and the full concentration-response curve gives the most information.

Are the targets likely to be the same as we have seen over the past few decades? In both academic research and industry it seems as though some of the problems are now more challenging. A reductionist approach can segregate every part of a linear pathway, but many of the pathways we are dealing with are not linear, they represent rather complex systems that do not provide simple signals, but rather they offer subtle modulating influences across a wide range of other pathways. The ghastly posters that sit on the walls of the scientist's office showing the multiplicity of pathways illustrate the problem, as do the review articles that can link every story together because it is now always possible to find a least one paper that supports the argument. So the field of systems biology is emerging to try to reintegrate and make sense of the pathways. In some ways there is nothing new here for pharmacology - understanding the therapeutic responses to glucocorticoids (see also Barnes; Buckingham, this issue) have provided something of a challenge for systems biology for many years.

So in this short section on research we have managed to squeeze in integrative physiology, functional genomics, chemical biology and systems biology. New words to describe a forward looking approach to areas that have been at the root of pharmacology for many years. We should recognise that the discovery of our area by molecular reductionists offers considerable opportunities for the pharmacological research of intact systems.

\section{Two sections - one too many}

If pharmacology is the study of drug action, then the divide between basic and clinical pharmacology must be arbitrary. Of course at one end of the spectrum the pharmacologist may only be using a drug to probe a basic mechanism of biology, and at the other end the clinical pharmacologist may be interested in the factors that determine why so many patients do not take the medicines prescribed. However, where is the line that divides clinical from basic? Is clinical pharmacology any study that involves humans? Or is it only research involving patients? Is a study of the effects of a potassium channel blocking drug in a rat basic pharmacology, whereas a similar study in humans clinical pharmacology? Many parts of the pharmaceutical industry have recognised the difficulties in the divide and have reorganised structures to incorporate experimental medicine into the discovery arm of its activities. In academia translational research and experimental medicine have recently become catchwords. The MRC has issued calls for proposals, and the Wellcome Trust and others have created a fund to establish new clinical research facilities. Clinical pharmacologists were some of the earliest proponents of experimental medicine and used small molecule probes to identify important pathways in human physiology.

Sidney Brenner has argued that 'man is the next model organism' and while his words relate to genetics and genomics, of course they apply to pharmacology as well. Basic pharmacology needs to be strong, well supported and free to explore areas irrespective of the clinical relevance. However, to thrive, clinical pharmacology needs to be a seamless continuation of basic pharmacology - there is no precise point at which one turns into the other. For an article on the future of pharmacology we rely a lot on the lessons of the past. In 1879 Brunton wrote "Before therapeutics can become a science, the physician must know the action of his drugs, just as the locksmith does of his keys, and since pharmacology is so young it is little wonder that medicine is yet only an art" (Lauder Brunton, 1880). This is as true today as it was then. 'Clinical pharmacology' is a useful shorthand for a clinical training programme, it does not describe a rigidly distinct research area within pharmacology. The future of pharmacology relies on removing arbitrary divisions between sections of the discipline, a discipline where the boundaries are already hard to define and possibly quite rightly blurred by the need to integrate numerous technologies and disciplines.

So in this section we have used experimental medicine and translational research - two more fashionable areas that mean that the opportunities for more clinically oriented pharmacological research are great. The formation of the U.K. Clinical Research Collaboration and the research networks funded from this should facilitate translation of research into clinical practice. The randomised controlled clinical trial of a drug does more than show the efficacy of the product, it can also prove the ultimate test of whether a biological pathway is causally important. Nevertheless, it is paramount to remember that in order to 'translate' it is necessary to have active, innovative, and probing basic science.

\section{Industry}

The relationship between academia and industry is nowhere more evident that in pharmacology. Most (nearly all) drugs emerge from industry, but most (nearly all) have had substantial academic input ranging from initial work on targets through to evaluation in the clinic. The relationship between academia and industry is also probably nowhere more ambivalent than in pharmacology. Collaboration is common, but some of the most fierce critics of industry are pharmacologists. This tension stems in part from the shared desire to generate new molecules of therapeutic benefit and the diverse 
remits which, on the one hand, are to market the successful product (industry), and, on the other, to regulate the product (often clinical pharmacologists).

The pharmaceutical industry needs academia to train its scientific workforce and a career in industry is a common goal for graduates and postgraduates in pharmacology. Movement between academia and industry at more senior research levels is increasingly common and benefits both parties. However, career advice for postdoctoral research fellows on opportunities provided by industry remains variable. The need for industry to increase its intake of clinician scientists provides an interesting insight into where academic pharmacology may be failing. The clinician scientists recruited tend to come from organ-based disciplines and not from within pharmacology/ clinical pharmacology. Furthermore, among this clinical group we do not yet know whether movement back and forth between academia and industry will be facilitated or even possible. Some of the new clinical training requirements may make this movement difficult.

Most of us were brought up to believe that small molecules are drugs and that peptides and biologicals did not provide good therapeutic options. Recent examples of the success of anti-TNF antibodies in rheumatoid arthritis (Feldmann \& Maini, 2003) and the development of enzyme replacement treatments, among others, show that this paradigm is changing. Moreover, use of peptides and protein therapeutics can provide a more rapid route to testing in man compared to the tortuous route often taken by conventional small drug molecules. The rapid progression to proof-of-concept in man will also allow more rapid assessment of the efficacy of such molecules in disease. However, many of the principles underlying the use of biologicals or indeed gene therapy are in essence pharmacological and these present new opportunities for basic and clinical pharmacologists. A major challenge for clinical pharmacologists in particular is to broaden the discipline to expand interests beyond small molecules and beyond the therapeutic areas of cardiovascular and respiratory disease (an aspect recognised widely in continental Europe). It is noteworthy that basic pharmacology is far more diverse in its subject area than clinical pharmacology in the U.K.

The pharmaceutical industry is strong in the U.K. and the government wants to retain that strong base. Recent reports highlight the need (Bioscience Innovation \& Growth Team, 2003) and the challenges (House of Commons Report on Pharmaceutical Industry). A thriving, forward-looking base in academic pharmacology is essential.

\section{Personalised medicines}

The genetic revolution has pushed to the forefront a concept that is at the very heart of pharmacology and therapeutics the right drug for the right target in the right patient and delivered at the right dose. In some areas such as cancer treatments, examples of specific tumour mutations that dictate the response to drugs is a reality. This will influence the nature of future clinical trials. The bigger challenge lies in understanding whether the same will be true for common germline genetic variation (polymorphisms) in the general population. Will common genetic variation influence response to treatment? Currently, the influence of common genetic variation on disease risk looks modest, with each individual functional variant providing a relative risk estimate in the order of 1.2. The complexity of turning this into a clinically meaningful risk score is such that attention has turned towards pharmaceuticals. Is drug failure due in individual patients due to genetic variation? Could drug safety be improved by taking into account individual genetic risk? Data on genetic variation influencing efficacy are sparse. There are some examples variants in genes involved in cholesterol synthesis absorption or transport may render individuals less susceptible to the lipid lowering effects of statins (Chasman et al., 2004), or variants in the P-glycoprotein transporter may influence drug resistance to antiepileptics (Siddiqui et al., 2003). However, the size of the effect is such that it is swamped by other factors such as the dose of drug (usually an effect can be obtained by increasing the dose or an unwanted effect may be diminished by decreasing the dose) or something as simple as adherence to treatment (not taking the medicine is a pretty good way to ensure it does not work). On the other hand, studies on susceptibility to unwanted effects are beginning to define variants that render individuals more at risk. It will be interesting to see whether genetic fingerprints that predict enhanced responsiveness or increased risk of unwanted effects can be established. This presents an exciting opportunity for pharmacology.

At the more fundamental mechanistic level, drugs are being used to tease out the phenotype associated with genetic variation. Given that each genetic variant has such a small effect on physiology, 'stressing' the physiology with a drug molecules is proving a useful way to identify extremes of phenotypic responses and thereby identify genetic influences. Using techniques such as analysis of 'Mendelian Randomisation' genetics will help determine which targets are likely to be important in disease causing pathways (Casas et al., 2005). On another level, by taking reductionist and integrated approaches, genetics and molecular pharmacology are beginning to unravel the enigmatic problem of drug receptor heterogeneity. The future promises new generations of drugs that could selectively target one subtype in a family of similar proteins to the therapeutic advantage of the patient while limiting commonly accepted and often debilitating side effects.

\section{Prescribing, policy and regulation}

Prescribers are changing. Getting onto the register to practice as a doctor brings rather few specific rights. In fact just about the only one is the right to prescribe medicines. However, nurses and pharmacists are also gaining these rights and this trend seems unlikely to reverse. Indeed, it makes sense to broaden the range of prescribers, and the challenge will be to ensure an appropriate pharmacological underpinning to ensure safe and effective use of medicines. Influencing policy on medicines has been a core activity of pharmacologists. The Committee on Safety of Medicines and the Medicines Commission have traditionally had strong representation from pharmacology, the MHRA and NICE are both led by clinical pharmacologists and the newly formed Human Medicines Commission has called for membership from pharmacologists and clinical pharmacologists. One only has to scan the pages of the major leading medical journals to see that issues of therapeutic effectiveness, cost-effectiveness and prescribing policy have become mainstream concerns. Many of those 
who have become involved in policy work and guiding the regulatory framework have performed so as individuals and, perhaps with a couple of exceptions, there has not been a thriving research base in these areas. The opportunities could not be greater and it would seem that this end of pharmacology should emerge as a major focus of research activity linking with epidemiologists, public health researchers, economists and social scientists. The emergence of these areas and the specific research questions around issues of adherence to drug treatments and clinical use are often used as an argument for maintaining a separate clinical section. The assumption behind this is that such areas will not be of interest to basic pharmacologists nor would they have a useful input to make. The basis for these assumptions may well be false.

\section{Teaching and training}

Research can and will adapt to new structures and new names. On the other hand, teaching and training across the range of areas of pharmacology need the discipline to exist as a discrete entity and require, above all, role models to inspire the next generation. At the undergraduate level the argument has been rehearsed many times and will be familiar to all - teach in organ-based themes and the organ-based specialist can teach about the drugs in their areas. After all, they are often the most familiar with those products. This experiment has taken place across medical schools and increasingly in undergraduate science courses. We do not yet have the data to know whether it is a failure, but there is certainly cause for concern. The knowledge about drugs among newly qualified doctors is not great (Dean et al., 2002), and concerns have been expressed at all levels about the ability of doctors to evaluate claims of therapeutic effect (House of Commons Report on Pharmaceutical Industry). A radical rethink of the way we teach about medicines is necessary to ensure that we equip the future generation of prescribers with the fundamental principles and ideas of pharmacology that underlie good prescribing practice.
At the science level we will need to be imaginative to capture the interest of the new students. The 'pick and mix' degree driven by concepts of 'choice' is part of most universities and we will need to make sure that we use the right words to describe our discipline that encompasses all the fashionable areas ranging from chemical biology to health policy. Ultimately, pharmacology ought to be attractive to students - it is both scientifically rigorous and has a clear relevance.

At the postgraduate level there are also opportunities relating to links with industry and joining up basic and clinical research. Rotational 4-year-Ph.D. studentships should allow exposure to the component parts that make up an understanding of the breadth of pharmacology. Greater mobility between academia and industry seems likely and the recent expansion in the number of clinician scientist posts should provide an opportunity to place high quality post-Ph.D. clinician scientists into the highest quality laboratories for further research training in basic and translational science.

\section{Conclusions}

So is it all gloom and doom? Far from it - the future of pharmacology ought to be bright. Which are the currently fashionable areas that funders wish to strengthen? Integrative biology/physiology, chemical biology/biological chemistry, pharmacogenetics, experimental medicine and systems biology. Look up the definitions of these various areas and an awful lot of it sounds like pharmacology. The horizon is definitely expanding and there will also be a need to understand at a molecular level how drugs are working. Where departments have survived they seem to be doing rather well. The notion of personalised pills has caught the imagination and, even before the input of genetics, is the essence of pharmacology - trying to understand how to make drugs precisely effective and safe and also establish how they work. If the future is 'pretty much like the present only longer', we need to get our act together now.

\section{References}

ABPI. (2005). Facts \& Statistics from the pharmaceutical industry. http://www.abpi.org.uk/statistics.

BIOSCIENCE INNOVATION \& GROWTH TEAM. (2003). Published by DTI.

CASAS, J.P., BAUTISTA, L.E., SMEETH, L., SHARMA, P. \& HINGORANI, A.D. (2005). Homocysteine and stroke: evidence on a causal link from mendelian randomisation. Lancet, 365, 194-196.

CHASMAN, D.I., POSADA, D., SUBRAHMANYAN, L., COOK, N.R., STANTON JR, V.P. \& RIDKER, P.M. (2004). Pharmacogenetic study of statin therapy and cholesterol reduction. JAMA, 291, 2821-2827.

DEAN, B., SCHACHTER, M., VINCENT, C. \& BARBER, N. (2002). Causes of prescribing errors in hospital inpatients: a prospective study. Lancet, 359, 1371-1378

FELDMANN, M. \& MAINI, R.N. (2003). Lasker Clinical Medical Research Award. TNF defined as a therapeutic target for rheumatoid arthritis and other autoimmune diseases. Nat. Med., 9, 1245-1250.

FURCHGOTT, R.F. \& ZAWADZKI, J.V. (1980). The obligatory role of endothelial cells in the relaxation of arterial smooth muscle by acetylcholine. Nature, 288, 373-376.

GALEN ON PHARMACOLOGY. (1995). Philosophy, history and medicine. In: Proceedings of the Fifth International Galen colloquium. ed. Debru, A. Amsterdam: Brill Academic Publishers.

HOUSE OF COMMONS REPORT ON PHARMACEUTICAL INDUSTRY. (1995). House of Commons Health Select Committee. Published by the Stationery Office Ltd.

LAUDER BRUNTON, T. (1880). Medicine Past and Present. London: MacMillan.

PIRMOHAMED, M., JAMES, S., MEAKIN, S., GREEN, C., SCOTT, A.K., WALLEY, T.J., FARRAR, K., PARK, B.K. \& BRECKENRIDGE, A.M. (2004). Adverse drug reactions as cause of admission to hospital: prospective analysis of 18,820 patients. $B M J, \mathbf{3 2 9}, 15-19$.

SCHEINDLIN, S. (2001). A brief history of pharmacology. Modern Drug Discovery, 4, 87-88.

SIDDIQUI, A., KERB, R., WEALE, M.E., BRINKMANN, U., SMITH, A., GOLDSTEIN, D.B., WOOD, N.W. \& SISODIYA, S.M. (2003). Association of multidrug reistance in epilepsy with a polymorphism in the drug-transporter gene ABCB1. N. Engl. J. Med., 348, $1480-1482$. 